\title{
Wavelet packets and their vanishing moments
}

\author{
NIKHIL KHANNA ${ }^{\dagger}$, VARINDER KUMAR AND S. K. KAUSHIK
}

\author{
Date of Receiving : $\quad$ : 26.05 .2017 \\ Date of $\mathrm{I}^{\text {st }}$ Revision : 21.07 .2017 \\ Date of II ${ }^{\text {nd }}$ Revision : 06.10 .2017 \\ Date of Acceptance : 21.10 .2017
}

\begin{abstract}
Sufficient condition for $\widehat{\omega}_{2 n+1}(\xi)$ and $m_{0}(\xi)$ and their first $q-1$ derivatives to vanish respectively at $\xi=0$ and $\xi=\pi$ has been obtained. A relationship between higher moments of $\omega_{2 n}$ and $\omega_{2 n+1}$ for wavelet packets associated with Haar wavelet has been given. Some results for Fourier transform of Hartley-like wavelet packets has been incurred and finally, sufficient conditions for higher vanishing moments of Hartley-like wavelet packets has been procured.
\end{abstract}

\section{Introduction}

In 1984, the combined effort of Grossmann and Morlet [5] directed to a complete mathematical study of the continuous wavelet transforms and their various applications. The wavelet theory has provided a new method for decomposing a function or a signal. In 1989, Mallat [10] and Meyer [13] presented the theory of multiresolution analysis. In 1996, Hernández and Weiss [6] studied higher vanishing moments of wavelets. Later in 2015, Khanna, Kumar and Kaushik $[7,8]$ studied vanishing moments of Hilbert transform of wavelets and give certain results to approximate the functions in $L^{2}(\mathbb{R})$. To fix the poor frequency localization of high frequency wavelet bases, its significant generalization was introduced by Coifman et al. [2] and thereby provide more adequate decomposition containing stationary and transient components. The wavelet packet method is a generalization of wavelet decomposition that offers a richer signal analysis. The wavelet packets can be used for numerous expansions of a given signal. Recently in 2016, Khanna, Kumar and Kaushik [9] introduced a notion of Hilbert transform of wavelet packets and Hartley-like wavelet packets. For various details related to wavelet packets, one may refer to $[2,6]$.

This paper deals with the study of wavelet packets. First, we give a sufficient condition under which $\widehat{\omega}_{2 n+1}(\xi)$ and low pass filter $m_{0}(\xi)$ and their first $q-1$ derivatives vanishes at $\xi=0$ and $\xi=\pi$ respectively. This is followed by a relationship between the $q^{\text {th }}$

2010 Mathematics Subject Classification. 42C40, 65T60.

Key words and phrases. Wavelet packets, Hartley-like wavelet packets, moments.

Communicated by: Sandra Saliani

${ }^{\dagger}$ Corresponding author. 